\title{
Familial cluster of asymptomatic COVID-19 cases in a First Nation community in Northern Saskatchewan, Canada
}

\author{
Shree Lamichhane1', Sabyasachi Gupta1', Grace Akinjobi', Nnamdi Ndubuka ${ }^{1,2 \star}$
}

Suggested citation: Lamichhane SR, Gupta S, Akinjobi G, Ndubuka N. Familial cluster of asymptomatic COVID-19 cases in a First Nation community in Northern Saskatchewan, Canada. Can Commun Dis Rep 2021;47(2):94-6. https://doi.org/10.14745/ccdr.v47i02a01

Keywords: COVID-19, asymptomatic, First Nation, Canada, familial cluster, transmission

\section{Introduction}

A novel coronavirus (severe acute respiratory syndrome coronavirus 2, SARS-CoV-2), causing a cluster of respiratory infections, initially appeared in Wuhan, China in December 2019. The outbreak spread rapidly around the world and, as of December 7, 2020, a total of 67,440,864 cases have been confirmed in 191 countries, resulting in 1,541,661 deaths. A wide range of coronavirus disease 2019 (COVID-19) symptoms has been reported, with symptoms ranging from mild to severe that may appear 2-14 days after exposure to the virus. Lately, it has been observed that the asymptomatic or presymptomatic cases make up what may be a large portion of all COVID-19 infections. If these cases cannot be identified and appropriately isolated for medical intervention, this could limit the effectiveness of transmission prevention measures.

We are reporting a familial cluster of COVID-19 cases that started with a paucisymptomatic case and led to two asymptomatic cases. In our familial cluster, five out of nine cases (55\%) were found to be presymptomatic at the time of testing, while two cases (22\%) remained asymptomatic throughout the course of the infection.

\section{Current situation}

Since the pandemic started, the province of Saskatchewan, Canada has reported 11,475 COVID-19 cases. Of these cases, 910 were from Northern Inter-Tribal Health Authority (NITHA) First Nations communities in the Northern Saskatchewan (http://www.nitha.com/). Given that the asymptomatic and presymptomatic persons are potential source of COVID-19 infection $(1,2)$, we are reporting a First Nations familial cluster from the Northern Saskatchewan where the infection started with a paucisymptomatic case and led to two asymptomatic cases. Increasingly, it is recognized that Indigenous determinants of health, such as overcrowding, poverty, impact of Indian
This work is licensed under a Creative Commons Attribution 4.0 International License.

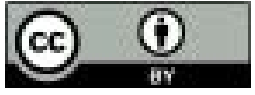

Affiliations

1 Northern Inter-Tribal Health Authority, Prince Albert, SK

2 School of Public Health, University of Saskatchewan, Saskatoon, SK

\section{*Correspondence:}

nndubuka@nitha.com residential school history, younger demographics, weak public health infrastructure, limited access to quality health services and higher rate of co-morbidities, can worsen disease outbreaks (3). Specifically, crowded housing conditions may result in ineffective physical distancing and inadequate infection control measures with increased likelihood of COVID-19 transmission. There is also an increased risk of poor mental health, hospitalizations and severe outcomes among those First Nationals individuals with immunocompromised and chronic disease conditions (4). As many First Nation communities are now being affected by COVID-19 outbreaks, this report also provides data necessary for the development and application of public health strategies within other First Nation communities.

Our index patient (20-29 years age group) acquired the infection from a close contact who returned to the community from an area of high transmission out-of-province and subsequently developed a mild symptom (rhinitis), which resolved within a few days. The index patient attended a family dinner two days later where further transmission appears to have occurred. After contact tracing, eight more cases were identified; three from the index's household and five from another household visited by the index patient (Figure 1). The exact timing of transmission exposure could not be ascertained because the 
persons with whom the index patient was in contact were living in overcrowded settings, and exposure was ongoing. No other possible exposures were identified that could link to these COVID-19 infection. All the COVID-19-positive patients and their close contacts were isolated in accordance with the provincial standards.

Patient 2 (10-19 years age group) and Patient 3 (30-39 years age group) from Household 1 developed very mild symptoms (loss of taste and smell) for two days; however, Patient 1 (40-49 years age group) did not developed any symptoms. From Household 2, Patient 6 became ill with a sore throat. Patient 5 (30-39 years age group, with a pre-existing chronic medical condition) reported the loss of taste and smell, followed by cough, shortness of breath and diarrhea. As the patient's condition worsened, this patient was hospitalized and recovered within two weeks. Patient 7 (an infant) became ill with a fever and cough; however, the patient's condition improved without medical intervention. Patient 8 (20-29 years age group), who initially tested negative for COVID-19 polymerase chain reaction testing, developed symptoms (wheeze and fever) at 12 days following exposure and was found to be COVID-19-positive on re-testing. Overall, three patients from Household 2 were found to be asymptomatic at time of testing; of them, one (Patient 4, 5-9 years age group) did not develop any symptoms throughout the isolation period.

In our familial cluster, five out of nine cases (55\%) were presymptomatic at time of testing while two cases (22\%) did not develop any symptoms throughout approximately two weeks of follow-up. Our index patient had only mild symptoms and was unaware of heightened COVID-19 risk status, which added to uncertainty and delayed the early detection and isolation. Despite these concerns, six out of nine cases developed only mild symptoms and recovered with minimal medical attention, highlighting the possibility of containment of COVID-19 cases outside the hospital with appropriate guidance and oversight. As rural communities can face different challenges around COVID-19 depending on where they located, each community and community members should assess their unique susceptibility and social vulnerability to COVID-19 and respond according to the public health measures. Relevant measures to prevent the COVID-19 community spread in these vulnerable communities would include avoiding non-essential travels outside the community and limiting interactions between different households.

\section{Conclusion}

Early detection and isolation of symptomatic COVID-19 patients with effective contact tracing investigations are an important disease containment strategy. As asymptomatic and presymptomatic transmission are biologically plausible $(1,2)$, such transmission could limit the effectiveness of control measures $(2,5,6)$. This case summary highlights the importance of early detection, contact tracing, testing of all close contactsregardless of the presence of symptoms-and preventive 14 days self-isolation of people returning to communities from high transmission areas to prevent asymptomatic spread in remote communities. It also highlights the need for low threshold for testing individuals with very mild symptoms in the 14 days post-return from high transmission areas. Transmissibility by asymptomatic or presymptomatic patients in the setting of crowded living conditions, such as those often seen in remote, northern and Indigenous communities, can contribute to higher transmission rates.

\section{Figure 1: Timeline of exposure to index and household cases in familial cluster}

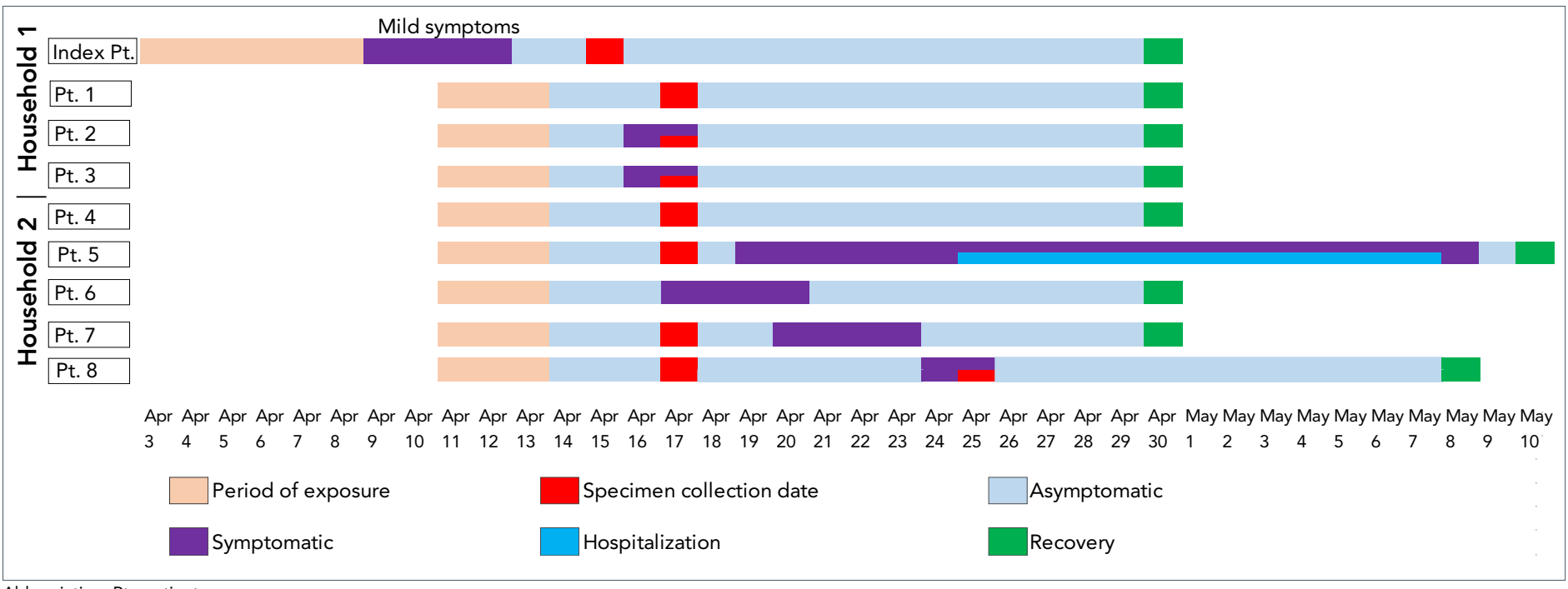

Abbreviation: Pt., patient 


\section{Authors' statement}

SRL — Literature review, writing-draft

SG — Data analysis, writing-draft

GA - Writing-review and editing

NN - Conception, design, data interpretation, critical review

\section{Competing interests}

None.

\section{Acknowledgements}

The authors gratefully acknowledge Northern Inter-Tribal Health Authority and partner communities for their hard work and contribution to this article.

\section{Funding}

None.

The content and view expressed in this article are those of the authors and do not necessarily reflect those of the Government of Canada.

\section{References}

1. Rothe $C$, Schunk M, Sothmann P, Bretzel G, Froeschl G, Wallrauch C, Zimmer T, Thiel V, Janke C, Guggemos W, Seilmaier M, Drosten C, Vollmar P, Zwirglmaier K, Zange S, Wölfel R, Hoelscher M. Transmission of 2019-nCoV infection from an asymptomatic contact in Germany. N Engl J Med 2020 Mar;382(10):970-1. DOI PubMed

2. Zhang J, Tian S, Lou J, Chen Y. Familial cluster of COVID-19 infection from an asymptomatic. Crit Care 2020 Mar;24(1):119. DOI PubMed

3. Richardson KL, Driedger MS, Pizzi NJ, Wu J, Moghadas SM. Indigenous populations health protection: a Canadian perspective. BMC Public Health 2012 Dec;12(1):1098. DOI PubMed

4. Schiavo R, May Leung M, Brown M. Communicating risk and promoting disease mitigation measures in epidemics and emerging disease settings. Pathog Glob Health 2014 Mar;108(2):76-94. DOI PubMed

5. Wei WE, Li Z, Chiew CJ, Yong SE, Toh MP, Lee VJ. Presymptomatic Transmission of SARS-CoV-2-Singapore, January 23-March 16, 2020. MMWR Morb Mort Wkly Rep. 2020;69(14):411-5. https://www.cdc.gov/mmwr/volumes/69/wr/ mm6914e1.htm

6. Tian S, Hu N, Lou J, Chen K, Kang $X$, Xiang Z, Chen $\mathrm{H}$, Wang D, Liu N, Liu D, Chen G, Zhang Y, Li D, Li J, Lian H, Niu S, Zhang L, Zhang J. Characteristics of COVID-19 infection in Beijing. J Infect. 2020;80(4):401-6. DOI PubMed 